Article

\title{
Dark Energy Problem, Physics of Early Universe and Some New Approaches in Gravity
}

\author{
Alexander Shalyt-Margolin \\ National Centre for Particle and High Energy Physics, Bogdanovich Street 153, Minsk 220040, Belarus; \\ E-Mail: a.shalyt@mail.ru or alexm@hep.by; Tel./Fax: +375-17-292-6075
}

Received: 7 September 2012; in revised form: 19 October 2012 / Accepted: 29 October 2012 /

Published: 2 November 2012

\begin{abstract}
The dark energy problem is studied based on the approach associated with the cosmological term in General Relativity that is considered as a dynamic quantity. It is shown that a quantum field theory of the Early Universe (Planck scales) and its limiting transition at low energy play a significant role. Connection of this problem with Verlinde's new (entropic) approach to gravity is revealed within the frame of such statement as well as the Generalized Uncertainty Principle (GUP) and Extended Uncertainty Principle (EUP). The implications from the obtained results are presented, and a more rigorous statement of the Concordance Problem in cosmology is treated.
\end{abstract}

Keywords: dark energy; deformation; entropic force; Planck scale

\section{Introduction}

At the present time the fact that the Dark Energy problem is basic for the fundamental physics is no longer a subject of much controversy. This is due to its clear statement with regard to the origin of the principal components of a modern Universe and the greatest part of its mass. Moreover, as the interfaces between different branches of theoretical physics (e.g., quantum field theory, gravity, and thermodynamics) become obliterated contributing to their interpenetration, various research methods are valuably enriched and supplemented.

Numerous works and review papers on Dark Energy problem have been published in the last 10-15 years [1-8], and a great number of approaches to this problem have been proposed: scalar field models (quintessence model, K-essence, tachyon field, phantom field, dilatonic, Chaplygin gas) [9-23], braneworld models [24-28], dynamic approaches to the cosmological constant $\Lambda$ [29], anthropic selection of $\Lambda[30]$. 
It might be well to mention the relevant new works on this subject that are primarily associated with the modified gravitation theory [31-33], etc. It is not worth considering all the research trends in this sphere, especially since a very detailed and rather remarkable review has been published recently [34].

It is clear that a dynamic aspect — the Dark energy changing in time-is one of the most important for this problem. Consequently, physics of the Early Universe, and particularly at Planck's scales, may be of great importance for better insight into the Dark Energy problem and for its solution.

In the last decade numerous works devoted to a Quantum Field Theory (QFT) at Planck's scale [35-41] have been published (of course, the author has no pretensions of being exhaustive in his references). This interest stems from the facts that (i) at these scales it is expected to reveal the effects of a Quantum Gravity (QG), and this still unresolved theory is intriguing all the researchers engaged in the field of theoretical physics; (ii) modern accelerators, in particular LHC, have the capacity of achieving the energies at which some QG effects may be exhibited.

Because in essence the Dark Energy is repulsive in nature, this problem is directly associated with gravity. In the last 15-20 years new very interesting approaches to gravity studies have been proposed [42-54], which may be divided into "thermodynamical" and "theoretical-informational" approaches. These approaches, contributing to better understanding of the problem, have a great impact on some theoretical physics studies including the Dark Energy problem.

As indicated by the title, in this work the above-mentioned three lines of research are interrelated.

\section{Dark Energy as Dynamical Vacuum Energy}

This section is based on the results obtained by the author in his previous works [55-57], and in particular [58].

The dark matter problem [59-61], along with the dark energy problem [1], is presently the basic problem in modern fundamental physics, astrophysics, and cosmology. Whereas for a nature of the first valid hypotheses have been accepted already [62], the dark energy still remains enigmatic [9-19]. However, it is the opinion of most researchers that dark energy represents the energy of the cosmic vacuum, its density being associated with the cosmological term.

In the last 15-20 years it has been shown that a density of the vacuum energy and the cosmological term, distinguished by the factor only, may be considered as dynamic quantities (for example, [29,63-71]. In the text below, we adhere to this point of view.

As is known, a direct calculation of the vacuum energy density $\rho_{v a c}$ results in the value that is higher than the experimental one by a factor of $10^{122}$. According to the typical reasoning that is already canonical, we have [72-74]:

$$
<\rho_{v a c}>=2 \int_{0}^{p_{\max }} d p \frac{4 \pi p^{2}}{(2 \pi \hbar)^{3}} \frac{\hbar \omega}{2}
$$

where $p_{\max } \sim P_{p l}$ is the momentum cut-off at Planck scales, because the General Relativity is valid right up to the Planck scales. Proceeding from $p=(\hbar \omega / c)$, we can obtain [72]

$$
<\rho_{v a c}>=\frac{\hbar \Omega^{4}}{8 \pi^{2} c^{3}}
$$


And then $\Omega$ is given by $\Omega_{p}$, where

$$
\hbar \Omega_{p}=E_{p}=\left(\frac{\hbar c^{5}}{G}\right)^{1 / 2}
$$

But it is known that $<\rho_{p}>$ is higher than the observable value $\rho_{v a c}^{o b s}[72,74]$ approximately by a factor of $10^{122}$.

Yet, taking the cosmological term as a dynamic quantity, we can write Equation (1) as

$$
<\rho_{v a c}(t)>=2 \int_{0}^{p(t)} d p \frac{4 \pi p^{2}}{(2 \pi \hbar)^{3}} \frac{\hbar \omega}{2}
$$

that in the very Early Universe, at the time close to the Planck's time $t_{p}$, turns to Equation (1) as follows:

$$
<\rho_{v a c}\left(t_{p}\right)>=2 \int_{0}^{P_{p l}} d p \frac{4 \pi p^{2}}{(2 \pi \hbar)^{3}} \frac{\hbar \omega}{2}
$$

However, it is known that the experimental value $\rho_{v a c}^{\text {obs }}$ is sooner determined by low momenta and energies (corresponding to large scales) and also by the infrared limit that is given by a radius of the measurable part of the Universe $R_{U n i v} \approx 10^{28} \mathrm{~cm}$. For such momenta by the uncertainty principle Equation (1) we have

$$
<\rho_{\text {vac }}\left(t_{\text {Univ }}\right)>=2 \int_{0}^{p_{\min }} d p \frac{4 \pi p^{2}}{(2 \pi \hbar)^{3}} \frac{\hbar \omega}{2}
$$

where $t_{\text {Univ }}$ is Universe life-time and $p_{\min }$ is "minimal" momentum.

As $R_{U n i v} / l_{p}=P_{p l} / p_{\min }=10^{61}$, then by analogy Equation (7) and in the infrared limit

$$
<\rho_{\text {vac }}\left(t_{\text {Univ }}\right)>=\frac{\hbar \Omega_{\text {min }}^{4}}{8 \pi^{2} c^{3}}
$$

where

$$
\hbar \Omega_{\min }=E_{\min }
$$

But in this case $\rho_{v a c}\left(t_{U n i v}\right)$ is lower than $\rho_{v a c}$ calculated from Equation (1) by a factor of $10^{244}$ rather than by a factor of $10^{122}$.

This result is easily obtained using the Uncertainty Principle for the pair of conjugate variables $(\Lambda, V)[75-78]$ :

$$
\Delta V \approx \frac{\hbar}{\Delta \Lambda}
$$

where $\Lambda$ is the dynamic cosmological term, and $V$ is the space-time volume that results from the Einstein-Hilbert action $S_{E H}$ [76]:

$$
\Lambda \int d^{4} x \sqrt{-g}=\Lambda V
$$

where Equation (10) is the term in $S_{E H}$.

In $[55,56]$ the Uncertainty Principle Equation (9) has been generalized at Planck scales up to the Generalized Uncertainty Principle

$$
\Delta V \approx \frac{\hbar}{\Delta \Lambda}+\alpha^{\prime} t_{p}^{2} \bar{V}^{2} \frac{\Delta \Lambda}{\hbar}
$$

where $\bar{V}$ is the spatial part of $V$ that, as is assumed, may be extracted explicitly. 
Equation (11) is of interest from the viewpoint of two limits [55,56]:

(1) IR - limit: $t \rightarrow \infty$

(2) UV - limit: $t \rightarrow t_{\text {min }}$.

In the case of IR-limit we have large volumes $\bar{V}$ and $V$ at low $\Delta \Lambda$. Because of this, the main contribution on the right-hand side of Equation (11) is made by the first term, as great $\bar{V}$ in the second term is damped by small $t_{p}$ and $\Delta \Lambda$. Thus, we derive at

$$
\lim _{t \rightarrow \infty} \Delta V \approx \frac{\hbar}{\Delta \Lambda}
$$

in accordance with Equation (9) and $\Lambda$ is a dynamic value fluctuating around zero.

And for the case (2) $\Delta \Lambda$ becomes significant

$$
\lim _{t \rightarrow t_{\text {min }}} \bar{V}=\bar{V}_{\text {min }} \sim \bar{V}_{p}=l_{p}^{3} ; \lim _{t \rightarrow t_{\text {min }}} V=V_{\text {min }} \sim V_{p}=l_{p}^{3} t_{p}
$$

As a result, we have

$$
\lim _{t \rightarrow t_{\min }} \Delta V=\frac{\hbar}{\Delta \Lambda}+\alpha_{\Lambda} V_{p}^{2} \frac{\Delta \Lambda}{\hbar}
$$

where the parameter $\alpha_{\Lambda}$ absorbs all the above-mentioned proportionality coefficients.

For Equation (14) $\Delta \Lambda \sim \Lambda_{p} \equiv \hbar / V_{p}=E_{p} / \bar{V}_{p}$.

It is easily seen that in this case $\Lambda \sim m_{p}^{4}$, in agreement with the value obtained using a standard (i.e., without super-symmetry and the like) quantum field theory [73,74]. Despite the fact that $\Lambda$ at Planck's scales (referred to as $\Lambda_{U V}$ ) (14) is also a dynamic quantity, it is not directly related to well-known $\Lambda$ Equations $(9,12)$ (called $\Lambda_{I R}$ ) because the latter, as opposed to the first one, is derived from Einstein's equations

$$
R_{\mu \nu}-\frac{1}{2} g_{\mu \nu} R=8 \pi G_{N}\left(-\Lambda g_{\mu \nu}+T_{\mu \nu}\right)
$$

However, Einstein's Equation (15) are not valid at the Planck scales and hence $\Lambda_{U V}$ may be considered as some high-energy generalization of the conventional cosmological constant, leading to $\Lambda_{I R}$ in the low-energy limit.

As $V \sim t l^{3} \sim l^{4}$, where $l$ - characteristic linear dimension $V$, it is directly inferred that in the infrared region $\Lambda_{I R}$ calculated for $l=R_{U n i v}$ is lower than $\Lambda_{U V}$ in $\sim R_{U n i v}^{4} / l_{p}^{4}=10^{244}$, that is in a perfect agreement with the result obtained by the direct calculations at the beginning of this Section.

In other words, a quantum field theory with UV-cutoff in the assumption that $\Lambda$ is a dynamic quantity gives no correct value for $\Lambda_{\text {observ }}$ as well.

In this way, similar to $[79,80]$, we obtain

$$
\Lambda_{D E}=\sqrt{\Lambda_{U R} \Lambda_{I R}}
$$

where $\Lambda_{D E}=\Lambda_{\text {observ }}$ is the cosmological term corresponding to the observable value.

As shown in [81], the Holographic Principle [82-86] is helpful in the solution of this problem. In addition, [55-57] demonstrate that variation of $\rho_{v a c}$ in time may be expressed adequately in terms of the deformation parameter $\alpha$ introduced in a quantum field theory at Planck's scales (Early Universe). This parameter is considered in greater detail in the following Section. 
In $[79,80]$ a similar expansion was performed for $\alpha(t)=\alpha_{I R}$ :

$$
\rho_{\mathrm{vac}}=\frac{1}{l_{p}^{4}}+\frac{1}{l_{p}^{4}}\left(\frac{l_{p}}{l_{\Lambda}}\right)^{2}+\frac{1}{l_{p}^{4}}\left(\frac{l_{p}}{l_{\Lambda}}\right)^{4}+\ldots=\frac{1}{l_{p}^{4}}\left(1+\alpha_{I R}+\alpha_{I R}^{2}+\ldots\right)
$$

Here $\rho_{\text {vac }}$ is understood as the quantity calculated for $l_{\Lambda}=l_{I R}=R_{U n i v}$ and $\alpha_{I R}$ corresponds to $l_{I R}$.

However, as shown previously, if the holographic principle is valid, this expansion has a more exact form

$$
<\rho(t)>=a_{1} \alpha(t)+a_{2} \alpha^{2}(t)+\ldots
$$

and $a_{1} \neq 0$.

\section{Quantum Theory of Early Universe}

In the last twenty years, the researchers have come to the understanding that studies of the Early Universe physics (extremely high Planck's energies) necessitate changes in the fundamental physical theories, specifically quantum mechanics and quantum field theory. Inevitably a fundamental length should be involved in these theories [87-91]. This idea has been first suggested by a string theory [92-94]. But it is still considered to be a tentative theory without the experimental status and merely an attractive model. However, the fundamental length has been involved subsequently in more simple and natural considerations [95]. The main approach to framing Quantum Mechanics with fundamental length (QMFL) and Quantum Field Theory with fundamental length (QFTFL) (or with Ultraviolet (UV) cutoff) is that associated with the Generalized Uncertainty Principle (GUP) [92-99]:

$$
\triangle x \geq \frac{\hbar}{\triangle p}+\alpha^{\prime} l_{p}^{2} \frac{\triangle p}{\hbar}
$$

with the corresponding Heisenberg algebra deformation produced by this principle [91,99].

Besides, in the works by the author [100-109] an approach to the construction of QMFL has been developed with the help of the deformed density matrix, the density matrix deformation in QMFL being a starting object called the density pro-matrix $\rho(\alpha)$ and deformation parameter (additional parameter)

$$
\alpha=l_{\min }^{2} / x^{2}
$$

where $x$ is the measuring scale, $l_{\min } \sim l_{p}$ and $0<\alpha \leq 1 / 4[100,101]$.

In this way, the deformation parameter mentioned in the end of the preceding Section is defined. Here the deformation is understood as an extension of a particular theory by inclusion of one or several additional parameters in such a way that the initial theory appears in the limiting transition [110].

The explicit form of the above-mentioned deformation gives the exponential ansatz [100-103,108,109]:

$$
\rho^{*}(\alpha)=\exp (-\alpha) \sum_{i} \omega_{i}|i><i|
$$

where all $\omega_{i}>0$ are independent of $\alpha$ and their sum is equal to 1.

Besides, the deformation parameter $\alpha$ is absolutely naturally represented as a ratio between the squared UV and IR limits

$$
\alpha=\left(\frac{U V}{I R}\right)^{2}
$$


where UV is fixed and IR is varying.

We can show that QFT parameters of deformations associated with GUP (i.e., corresponding deformed Heisenberg algebra) may be expressed in terms of the parameter $\alpha[55,56]$.

Then

$$
[\vec{x}, \vec{p}]=i \hbar\left(1+\beta^{2} \vec{p}^{2}+\ldots\right)=i \hbar\left(1+a_{1} \alpha+a_{2} \alpha^{2}+\ldots\right)
$$

where $a_{1}, a_{2}, \ldots$ are some numerical factors.

The deformation parameter $\alpha$ has very interesting features:

(1) obviously, it is small and dimensionless;

(2) its variation is rapid at high energies and slow at low energies;

(3) it has ambiguous physical meaning, on the one hand being an inverse entropy of the black hole with a corresponding radius up to a certain numerical factor, and on the other hand being a minimal area multiplied by the square of the corresponding curvature;

(4) it is useful in solving of some problems of the black hole physics including the Information Paradox problem $[103,104,108]$ and, as mentioned in the preceding section, it gives an adequate expression for a density of the vacuum energy in a dynamic approach.

\section{New Approaches to Gravity and Possibility for Their High-Energy Generalization}

As noted in the introduction section, the recently developed methods [42-54] have contributed to better insight into gravity and the associated problems. Proceeding from a study of the literature data, based on the author's previous results, and assuming the cosmological term to be a dynamic quantity, we are interested in the generalization, if possible, of the above-mentioned methods to high (Planck) energies). A possibility of such generalization has been studied by the author in [57,58]. Specifically, in the works referenced the high-energy $\alpha$-deformation of Einstein equations has been examined for horizon spaces based on representation in the form of a thermodynamic identity ([53], formula (119))

$$
\underbrace{\frac{\hbar c f^{\prime}(a)}{4 \pi}}_{k_{B} T} \underbrace{\frac{c^{3}}{G \hbar} d\left(\frac{1}{4} 4 \pi a^{2}\right)}_{d S} \underbrace{-\frac{1}{2} \frac{c^{4} d a}{G}}_{-d E}=\underbrace{\operatorname{Pd}\left(\frac{4 \pi}{3} a^{3}\right)}_{P d V}
$$

considering a static, spherically symmetric horizon in the space-time described by the metric

$$
d s^{2}=-f(r) c^{2} d t^{2}+f^{-1}(r) d r^{2}+r^{2} d \Omega^{2}
$$

The horizon location is given by a simple zero of the function $f(r)$, at $r=a, f(a)=0$ and $f^{\prime}(a) \neq 0$; $T$ is the corresponding temperature ([53], eq.(116)); $P=T_{r}^{r}$ is the trace of the momentum-energy tensor and radial pressure.

First, in [57,58] $\alpha$-deformation was derived for Equation (24) replacement of $a$ by $\alpha$ ) at known energies: $\alpha \ll 1 / 4$. In this case $P=T_{r}^{r}$ in the right-hand side of Equation (24) is also apparently dependent on $\alpha: P=P(\alpha)$. Then in Equation (24) a limiting transition is made to $\alpha \rightarrow 1 / 4$ but now by replacement of all the fundamental quantities $T(\alpha), S(\alpha), E(\alpha), V(\alpha), \ldots$, in this equation by the corresponding high-energy ones, with quantum corrections of the deformation due to GUP Equation (19) $T_{G U P}(\alpha), S_{G U P}(\alpha), E_{G U P}(\alpha), V_{G U P}(\alpha), \ldots$ (for example [111,112]). In so doing two different cases are 
possible, one of which may be considered within the scope of equilibrium thermodynamics and the other within non-equilibrium thermodynamics.

(I) Equilibrium case. On going to $\alpha \rightarrow 1 / 4$, the general form of (24) remains invariable, whereas the high-energy $\alpha$-deformation of Equation (24) takes the following form ([57], formula (50)):

$$
k_{B} T_{G U P}(\alpha) d S_{G U P}(\alpha)-d E_{G U P}(\alpha)=P(\alpha) d V_{G U P}(\alpha)
$$

(II) Non-equilibrium case. In this case the equality in Equation (26) is upset by the dynamic cosmological term $\Lambda=\Lambda(\alpha)$. Next we go to the case of non-equilibrium thermodynamics. Assuming $\hbar=c=1$, we can write a non-equilibrium analog for Equation (26) as ([57], formula (53))

$$
k_{B} T_{G U P}(\alpha) d S_{G U P}(\alpha)-d E_{G U P}(\alpha)=P(\alpha) d V_{G U P}(\alpha)-G \Lambda(\alpha)
$$

which is in a complete agreement with the results in Section 2 of the present paper: in the Early Universe (at great $\alpha$, i.e., for $\alpha \approx 1 / 4$ ) the cosmological term $\Lambda(\alpha)$ has a high value, in line with inflation models [113,114]. At low energies, or for $\alpha \approx 0, \Lambda(\alpha)$ is small. In Section 2, it is demonstrated that, within the scope of the holographic principle, in series expansion of $\Lambda(\alpha)$ in terms of $\alpha$ the leading term is to the first power enabling one to account for a factor of $10^{122}$ discrepancy between the theoretical value derived from QFT and the observed value of $\Lambda$.

It is clear that the limiting transition to lower energies from Equation (27), i.e., to small values of $\alpha$, is given to a high accuracy by Equation (26).

In the foregoing it is not improbable that $P(\alpha)=0$. In this case in Equation (27) we get the de Sitter space, all the previous calculations being valid.

Solutions for both Equations (26) and (27) may be obtained as a series expansion in terms of $\alpha$. However, it should be noted that such an expansion at high energies should be close to $\alpha \approx 1 / 4$ and on going to the low-energy limit to $\alpha \approx 0$.

More details may be found in [57]. So, as indicated in [57], the dynamic cosmological term is a measure of deviation from the thermodynamic identity (the first law of thermodynamics) of the high-energy deformation of Einstein equations for horizon spaces in their thermodynamic interpretation.

\section{Comments and Conclusion}

In the previous section the case of the high-energy deformation of gravity has been considered for horizon spaces based on GUP or QFT with a minimal length, i.e., with the UV-cutoff.

At the same time, to study the region of low energies, we have to consider QFT with a minimal momentum (or IR-cutoff) [111,112].

As shown in [111], a minimal momentum arises from the Extended Uncertainty Principle (EUP) as follows:

$$
\Delta x_{i} \Delta p_{j} \geq \hbar \delta_{i j}\left[1+\beta^{2} \frac{\left(\Delta x_{i}\right)^{2}}{l^{2}}\right]
$$

where $l$ is the characteristic, large length scale $l \gg l_{p}$ and $\beta$ is a dimensionless real constant on the order of unity [111]. From Equation (28) we get an absolute minimum in the momentum uncertainty

$$
\Delta p_{i} \geq \frac{2 \hbar \beta}{l}
$$


Naturally, QFT with EUP (just as QFT with GUP) is a deformation of well-known QFT. As shown in [115], this deformation ("EUP-deformation") of QFT may be also described in terms of the parameter $\alpha$, i.e., in fact it is the $\alpha$-deformation.

Moreover, in [115] it is demonstrated that the General Relativity deformation in the presence of EUP or EUP-deformation (as well as GUP-deformation) is in essence the $\alpha$-deformation provided the corresponding Einstein's equations (similar to the case of GUP-deformation) have a thermodynamic interpretation. From this viewpoint, the parameter $\alpha$ is thought to be universal. It seems that some hints to a nature of such deformation may be found in the works devoted to the infrared modification of gravity [116-119].

In [54] gravity has been considered as a (non-fundamental) secondary interaction caused by changes in entropy on the holographic screen, which is called the "Entropic Force". As shown in [120], within the scope of GUP, the results from [54] may be extended to the ultraviolet region (Planck's energies) and the corresponding quantum corrections to Einstein Equations may be obtained. These results have been derived in terms of the parameter $\alpha$, the appropriate UV-modification of the results from [54] with $\alpha$ being the deformation, at least within the scope of GUP.

The result from [54] has been obtained in the assumption that the cosmological term $\Lambda=0$ is equal to zero. This is reasonable as in [54] for mass in the asymptotically flat space-time [121,122] the Komar's formula initially taken in the assumption of $\Lambda=0$ has been used. However, in [123] this Komar's formula for mass (Komar's integral) was generalized to the case $\Lambda \neq 0$.

In ([120], formulas (33),(34)), with the use of the formulae derived in [123], the principal result from [54] has been generalized to the case of the dynamic cosmological term $\Lambda=\Lambda[\alpha(t)] \neq 0$ in line with the considerations in Section 2 of the present paper but given from the viewpoint of gravity.

Concordance Problem in Cosmology in the general form is formulated as follows: why in the modern epoch densities of the Dark Energy and Matter (the Dark Matter including) are approximately the same, at least on the order of magnitude?

Based on the approach presented in this paper, the answer may be the following: this is possibly due to the fact that presently in a power series expansion of the corresponding densities $\rho_{\mathrm{DE}}=\rho_{\mathrm{vac}}$ and $\rho_{\text {matter }}$ in terms of the small parameter $\alpha$, the leading terms are of the same power. In this case, further studies of the problem at hand will be reduced to examination of the corresponding factors of this power.

\section{References}

1. Perlmutter, S.; Aldering, G.; Goldhaber, G.; Knop, R.A.; Nugent, P.; Castro, P.G.; Deustua, S.; Fabbro, S.; Goobar, A.; Groom, D.E.; et al. Measurements of Omega and Lambda from 42 high redshift supernovae. Astrophys. J. 1999, 517, 565-586.

2. Riess, A.G.; Filippenko, A.V.; Challis, P.; Clocchiattia, A.; Diercks, A.; Garnavich, P.M.; Gilliland, R.L.; Hogan, C.J.; Jha, S.; Kirshner, R.P.; et al. Observational evidence from supernovae for an accelerating universe and a cosmological constant. Astron. J. 1998, 116, 1009-1038.

3. Riess, A.G. ; Kirshner, R.P.; Schmidt, B.P.; Jha, S.; Challis, P.; Garnavich, P.M.; Esin, A.A.; Carpenter, C.; Grashius, R.; Schild, R.E.; et al. BV RI light curves for 22 type Ia supernovae. Astron. J. 1999, 117, 707-724. 
4. Sahni, V.; Starobinsky, A.A. The case for a positive cosmological Lambda term. Int. J. Mod. Phys. D 2000, 9, 373-397.

5. Carroll, S.M. The cosmological constant. Living Rev. Rel. 2001, 4, 1-50.

6. Padmanabhan, T. Cosmological constant: The weight of the vacuum. Phys. Rept. 2003, 380, 235-320.

7. Padmanabhan, T. Dark energy: The cosmological challenge of the millennium. Curr. Sci. 2005, 88, 1057-1071.

8. Peebles, P.J.E.; Ratra, B. The cosmological constant and dark energy. Rev. Mod. Phys. 2003, 75, 559-606.

9. Ratra, B.; Peebles, J. Cosmological consequences of a rolling homogeneous scalar field. Phys. Rev. D 1988, 37, 3406-3422.

10. Caldwell, R.R.; Dave, R.; Steinhardt, P.J. Cosmological imprint of an energy component with general equation of state. Phys. Rev. Lett. 1998, 80, 1582-1585.

11. Armendariz-Picaon, C.; Damour, T.; Mukhanov, V. K-inflation. Phys. Lett. B 1999, 458, 209-218.

12. Garriga, J.; Mukhanov, V. Perturbations in k-inflation. Phys. Lett. B 1999, 458, 219-225.

13. Padmanabhan, T. Accelerated expansion of the universe driven by tachyonic matter. Phys. Rev. D 2002, 021301.

14. Bagla, J.S.; Jassal, H.K.; Padmanabhan, T. Cosmology with tachyon field as dark energy. Phys. Rev. D 2003, 063504.

15. Abramo, L.R.W.; Finelli, F. Cosmological dynamics of the tachyon with an inverse power-law potential. Phys. Lett. B 2003, 575, 165-171.

16. Aguirregabiria, J.M.; Lazkoz, R. Tracking solutions in tachyon cosmology. Phys. Rev. D 2004, 123502.

17. Guo, Z.K.; Zhang, Y.Z. Cosmological scaling solutions of the tachyon with multiple inverse square potentials. J. Cosmol. Astropart. Phys. 2004, 0408, 010.

18. Copeland, E.J.; Garousi, M.R.; Sami, M.; Tsujikawa, S. What is needed of a tachyon if it is to be the dark energy? Phys. Rev. D 2005, 043003.

19. Sahni, V.; Shtanov, Y. Brane world models of dark energy. J. Cosmol. Astropart. Phys. 2003, $0311,014$.

20. Elizalde, E.; Nojiri, S.; Odintsov, S.D. Late-time cosmology in (phantom) scalar-tensor theory: Dark energy and the cosmic speed-up. Phys. Rev. D 2004, 043539.

21. Gasperini, M.; Piazza, F.; Veneziano, G. Quintessence as a run-away dilaton. Phys. Rev. $D$ 2002, 023508.

22. Damour, T.; Piazza, F.; Veneziano, G. Runaway dilaton and equivalence principle violations. Phys. Rev. Lett. 2002, 081601.

23. Kamenshchik, A.Y.; Moschella, U.; Pasquier, V. An alternative to quintessence. Phys. Lett. $B$ 2001, 511, 265-268.

24. Randall, L.; Sundrum, R. A large mass hierarchy from a small extra dimension. Phys. Rev. Lett. 1999, 83, 4690-4693. 
25. Nilles, H.P.; Papazoglou, A.; Tasinato, G. Selftuning and its footprints. Nucl. Phys. B 2004, 677, 405-429.

26. Aghababaie, Y.; Burgess, C.P.; Parameswaran, S.L.; Quevedo, F. Towards a naturally small cosmological constant from branes in 6D supergravity. Nucl. Phys. B 2004, 680, 389-414.

27. Burgess, C.P. Towards a natural theory of dark energy: Supersymmetric large extra dimensions. AIP Conf. Proc. 2005, 743, 417-449.

28. Arefeva, I.Y.; Dragovic, B.G.; Volovich, I.V. The extra timelike dimensions lead to a vanishing cosmological constant. Phys. Lett. B 1986, 177, 357-362.

29. Mukohyama, S.; Randall, L. A dynamical approach to the cosmological constant. Phys. Rev. Lett. 2004, 211302.

30. Garriga, J.; Vilenkin, A. On likely values of the cosmological constant. Phys. Rev. D 2000, 083502.

31. Elizalde, E.; Nojiri, S.; Odintsov, S.D.; Saez-Gomez, D. Unifying inflation with dark energy in modified F(R) horava-lifshitz gravity. Eur. Phys. J. C 2010, 70, 351-361,

32. Capozziello, S.; Matsumoto, J.; Nojiri, S.; Odintsov, S.D. Dark energy from modified gravity with lagrange multipliers. Phys. Lett. B 2010, 693, 351-361.

33. Astashenok, A.V.; Nojiri, S.; Odintsov, S.D.; Scherrer, R.J. Scalar dark energy models mimicking $\Lambda$ CDM with arbitrary future evolution. Phys .Lett. B 2012, 713, 145-153.

34. Bamba, K.; Capozziello, S., Nojiri, S.; Odintsov, S.D. Dark energy cosmology: The equivalent description via different theoretical models and cosmography tests. 2012, arXiv: 1205.3421.

35. Klinkhamer, F.R. Fundamental length scale of quantum spacetime foam. JETP Lett. 2007, 86, 2167-2180.

36. Amelino-Camelia, G.; Smolin, L. Prospects for constraining quantum gravity dispersion with near term observations. Phys. Rev. D 2009, 084017.

37. Gubitosi, G.; Pagano, L.; Amelino-Camelia, G.; Melchiorri, A.; Cooray, A. A constraint on planck-scale modifications to electrodynamics with CMB polarization data. J. Cosmol. Astropart. Phys. 2009, 0908, 021.

38. Amelino-Camelia, G. Building a case for a planck-scale-deformed boost action: The planck-scale particle-localization limit. Int. J. Mod. Phys. D 2005, 14, 2167-2180.

39. Hossenfelder, S.; Bleicher, M.; Hofmann, S.; Ruppert, J.; Scherer, S.; Stocker, H. Signatures in the planck regime. Phys. Lett. B 2003, 575, 85-99.

40. Hossenfelder, S. Running coupling with minimal length. Phys. Rev. D 2004, 70, 105003.

41. Hossenfelder, S. Self-consistency in theories with a minimal length. Class. Quant. Grav. 2006, $23,1815-1821$.

42. Jacobson, T. Thermodynamics of space-time: The einstein equation of state. Phys. Rev. Lett. 1995, 75, 1260-1263.

43. Padmanabhan, T. A new perspective on gravity and the dynamics of spacetime. Int. J. Mod. Phys. 2005, D14, 2263-2270.

44. Padmanabhan, T. The holography of gravity encoded in a relation between entropy, horizon area and action for gravity. Gen. Rel. Grav. 2002, 34, 2029-2035. 
45. Padmanabhan, T. Holographic gravity and the surface term in the einstein-hilbert action. Braz. $J$. Phys. 2005, 35, 362-372.

46. Padmanabhan, T. Gravity: A new holographic perspective. Int. J. Mod. Phys. D 2006, 15, 1659-1676.

47. Mukhopadhyay, A.; Padmanabhan, T. Holography of gravitational action functionals. Phys. Rev. D 2006, 124023.

48. Padmanabhan, T. Dark energy and gravity. Gen. Rel. Grav. 2008, 40, 529-564.

49. Padmanabhan, T.; Paranjape, A. Entropy of null surfaces and dynamics of spacetime. Phys. Rev. D 2007, 064004.

50. Padmanabhan, T. Gravity as an Emergent Phenomenon: A Conceptual Description. 2007, arXiv: 0706.1654v1 [gr-qc].

51. Padmanabhan, T. Gravity and the thermodynamics of horizons. Phys. Rept. 2005, 406, 49-125.

52. Paranjape, A.; Sarkar, S.; Padmanabhan, T. Thermodynamic route to field equations in lancos-lovelock gravity. Phys. Rev. D 2006, 104015.

53. Padmanabhan, T. Thermodynamical aspects of gravity: New insights. Rep. Prog. Phys. 2010, 046901.

54. Verlinde, E. On the origin of gravity and the laws of newton. J. High Energy Phys. 2011, $1104,029$.

55. Shalyt-Margolin, A.E. Entropy in the present and early universe and vacuum energy. AIP Conf. Proc. 2010, 1205, 160-167.

56. Shalyt-Margolin, A.E. Entropy in the present and early universe: New small parameters and dark energy problem. Entropy 2010, 12, 932-952.

57. Shalyt-Margolin, A.E. Quantum theory at planck scale, limiting values, deformed gravity and dark energy problem. Int. J. Mod. Phys. D 2012, 1250013.

58. Shalyt-Margolin, A.E. Quantum theory at planck scale, dynamical cosmological term and deformed gravity. Int. J. Theory Math. Phys. 2011, 1, 1-12.

59. Sciama, D.W. Cosmology, galactic astronomy and elementary particle physics. Proc. R. Soc. Lond. A 1984, 394, 1-17.

60. Bowyer, S.; Korpela, E.J.; Edelstein, J.; Lampton, M.; Morales, C.; Perez-Mercader, J.; Gomez, J.F.; Trapero, J. Evidence against the sciama model of radiative decay of massive neutrinos. Astrophys. J. 1999, doi: 10.1086/307990.

61. Turner, M.S. Dark matter in the universe. Phys. Scripta 1991, T36, 167-182.

62. Hooper, D. TASI 2008 lectures on dark matter. 2009, arXiv: 0901.4090 [hep-ph].

63. Bertolami, O.; Cim. N. Time dependent cosmological term. Nuovo Cim. B 1986, 93, 36-42.

64. Carvalho, J.C.; Lima, J.A.S.; Waga, I. On the cosmological consequences of a time dependent lambda term. Phys. Rev. D 1992, 46, 2404-2407.

65. Chimento, L.P.; Pavon, D. Inhomogeneous universe models with varying cosmological term. Gen. Rel. Grav. 1998, 30, 643-651.

66. Harko, T.; Mak, M.K. Particle creation in cosmological models with varying gravitational and cosmological "constants". Gen. Rel. Grav. 1999, 31, 849-862.

67. Carneiro, S. Decaying lambda cosmology with varying G. 2003, arXiv: gr-qc/0307114. 
68. Aldrovandi, R.; Beltran-Almeida, J.P.; Pereira, J.G. Time-varying cosmological term: Emergence and fate of a FRW comments. Grav. Cosmol. 2005, 11, 277-283.

69. Cai, R.-G.; Hu, B.; Zhang, Y. Holography, UV/IR Relation, causal entropy bound and dark energy. Commun. Theory Phys. 2009, 51, 954-960.

70. Shapiro, I.L.; Sola, J. Can the cosmological "constant" run? - It may run. 2008, arXiv: 0808.0315 [hep-th].

71. Richard, T.H.; Pilling, T. Dark entropy. 2008, arXiv: 0806.1277.

72. O'Connell, R.F. The expansion of the universe and the cosmological constant problem. Phys. Lett. A 2007, 366, 177-178.

73. Zel'dovich, Y.B. The cosmological constant and the theory of elementary particles. Sov. Phys. Uspehi 1968 11, 381-393.

74. Weinberg, S. The cosmological constant problem. Rev. Mod. Phys. 1989, 61, 1-23.

75. Jejjala, V.; Kavic, M.; Minic, D. Time and M-theory. Int. J. Mod. Phys. A 2007, 22, 3317-3405.

76. Jejjala, V.; Kavic, M.; Minic, D. Fine structure of dark energy and new physics. Adv. High Energy Phys. 2007, 21586.

77. Jejjala, V.; Minic, D. Why there is something so close to nothing: Towards a fundamental theory of the cosmological constant. Int. J. Mod. Phys. A 2007, 22, 1797-1818.

78. Jejjala, V.; Minic, D.; Tze, C.-H. Toward a background independent quantum theory of gravity. Int. J. Mod. Phys. D 2004, 13, 2307-2314.

79. Padmanabhan, T. Darker Side of the Universe ... and the Crying Need for Some Bright Ideas! In Proceedings of the 29th International Cosmic Ray Conference, Pune, India, 3-10 August 2005; pp. 47-62.

80. Padmanabhan, T. Dark Energy: Mystery of the Millennium. American Institute of Physics Conference Proceedings 2006, 861, 858-866.

81. Balazs, C.; Szapudi, I. Naturalness of the vacuum energy in holographic theories. 2006, arXiv: hep-th/0603133.

82. Hooft, G.T. Dimensional reduction in quantum gravity. Essay dedicated to Abdus Salam. 2009, arXiv: gr-qc/9310026.

83. Hooft, G.T. The holographic principle. 2001, arXiv: hep-th/0003004.

84. Susskind, L. The world as a hologram. J. Math. Phys. 1995, 36, 6377-6396.

85. Bousso, R. The holographic principle. Rev. Mod. Phys 2002, 74, 825-874.

86. Bousso, R. A covariant entropy conjecture. J. Cosmol. Astropart. Phys. 1999, 07, 004.

87. Garay, L. Quantum gravity and minimum length. Int. J. Mod. Phys. A 1995, 10, 145-166.

88. Ahluwalia, D.V. Wave particle duality at the Planck scale: Freezing of neutrino oscillations. Phys. Lett. 2000, A275, 31-35.

89. Ahluwalia, D.V. Interface of gravitational and quantum realms. Mod. Phys. Lett. 2002, A17, 1135-1146.

90. Maggiore, M. A generalized uncertainty principle in quantum gravity. Phys. Lett. B 1993, B304, 65-69.

91. Maggiore, M. The algebraic structure of the generalized uncertainty principle. Phys. Lett. B 1993, $319,83-86$. 
92. Veneziano, G. A stringy nature needs just two constants. Europhys. Lett. 1986, 2, 199-211.

93. Amati, D.; Ciafaloni, M.; Veneziano, G. Can space-time be probed below the string size? Phys. Lett. B 1989, 216, 41-47.

94. Witten, E. Reflections on the fate of spacetime. Phys. Today 1996, 49, 24-28.

95. Adler, R.J.; Santiago, D.I. On gravity and the uncertainty principle. Mod. Phys. Lett. A 1999, 14, 1371-1378.

96. Scardigli, F. Generalized uncertainty principle in quantum gravity from micro-black hole Gedanken experiment. Phys. Lett. B 1999, 452, 39-44.

97. Bambi, C. A revision of the generalized uncertainty principle. Class. Quant. Grav. 2008, 25, 105003.

98. Bolen, B.; Cavaglia, M. (Anti-)de Sitter black hole thermodynamics and the generalized uncertainty principle. Gen. Rel. Grav. 2005, 37, 1255-1263.

99. Kempf, A.; Mangano, G.; Mann, R.B. Hilbert space representation of the minimal length uncertainty relation. Phys. Rev. D 1995, 52, 1108-1118.

100. Shalyt-Margolin, A.E.; Suarez, J.G. Quantum mechanics of the early universe and its limiting transition. 2003, arXiv: gr-qc/0302119.

101. Shalyt-Margolin, A.E.; Suarez, J.G. Quantum mechanics at Planck's scale and density matrix. Int. J. Mod. Phys. D 2003, 12, 1265-1278.

102. Shalyt-Margolin, A.E.; Tregubovich, A.Y. Deformed density matrix and generalized uncertainty relation in thermodynamics. Mod. Phys. Lett. A 2004, 19, 71-82.

103. Shalyt-Margolin, A.E. Nonunitary and unitary transitions in generalized quantum mechanics, new small parameter and information problem solving. Mod. Phys. Lett. A 2004, 19, 391-404.

104. Shalyt-Margolin, A.E. Pure states, mixed states and Hawking problem in generalized quantum mechanics. Mod. Phys. Lett. A 2004, 19, 2037-2045.

105. Shalyt-Margolin, A.E. The universe as a nonuniform lattice in finite volume hypercube. I. Fundamental definitions and particular features. Int. J. Mod. Phys. D 2004, 13, 853-864.

106. Shalyt-Margolin, A.E. The universe as a nonuniform lattice in the finite-dimensional hypercube. II. Simple cases of symmetry breakdown and restoration. Int. J. Mod. Phys. A 2005, 20, 4951-4964.

107. Shalyt-Margolin, A.E.; Strazhev, V.I. The Density Matrix Deformation in Quantum and Statistical Mechanics in Early Universe. In Proceedings of the Sixth International Symposium "Frontiers of Fundamental and Computational Physics, Udine, Italy, 26-29 September,2004; Sidharth, B.G., Honsell, F., de Angelis, A., Eds.; Springer: Berlin/Heidelberg, Germany, 2006; pp. 131-134.

108. Shalyt-Margolin, A.E. The Density Matrix Deformation in Physics of the Early Universe and Some of Its Implications. In Quantum Cosmology Research Trends, Reimer, A., Ed.; Nova Science Publishers Inc.: Hauppauge, NY, USA, 2005; pp. 49-91.

109. Shalyt-Margolin, A.E. Deformed density matrix and quantum entropy of the black hole. Entropy 2006, 8, 31-43.

110. Faddeev, L. Mathematical view on evolution of physics. Priroda 1989, 5, 11-18.

111. Park, M.-I. The generalized uncertainty principle in (A)dS space and the modification of hawking temperature from the minimal length. Phys. Lett. B 2008, 659, 698-702. 
112. Kim, W.; Son, E.J.; Yoon, M. Thermodynamics of a black hole based on a generalized uncertainty principle. J. Cosmol. Astropart. Phys. 2008, 08, 035.

113. Kolb, E.; Turner, M. The Early Universe ; Addison Wesley: Reading, UK, 1990.

114. Baumann, D. TASI lectures on inflation. 2009, ArXiv: 0907.5424.

115. Shalyt-Margolin, A.E. Deformed quantum field theory, thermodynamics at low and high energies, and gravity. II. Deformation parameter. Int. J. Theory Math. Phys. 2012, 2, 41-50.

116. Dvali, G. Infrared modification of gravity. 2004, arXiv: hep-th/0402130.

117. Patil, S.P. Degravitation, inflation and the cosmological constant as an afterglow. J. Cosmol. Astropart. Phys. 2009, 0901, 017.

118. Rubakov, V.A.; Tinyakov, P.G. Infrared-modified gravities and massive gravitons. Phys. Usp 2008, 51, 123, 759-792.

119. Nikiforova, V.; Randjbar-Daemi, S.; Rubakov, V. Infrared modified gravity with dynamical torsion. Phys. Rev. D 2009, 80, 124050.

120. Shalyt-Margolin, A.E. Probable entropic nature of gravity in ultraviolet and infrared limits. I.Ultraviolet case. 2012, arXiv:1205.6988.

121. Komar, A. Covariant conservation laws in general relativity. Phys. Rev. 1959, 113, 934-936.

122. Wald, R.M. General Relativity; The University Chicago Press: Chicago, IL, USA, 1984.

123. Magnon, A. On komar integrals in asymptotically anti de sitter spacetimes. J. Math. Phys. 1985 , 26, 3112-3117.

(c) 2012 by the author; licensee MDPI, Basel, Switzerland. This article is an open access article distributed under the terms and conditions of the Creative Commons Attribution license (http://creativecommons.org/licenses/by/3.0/). 